\title{
An Automated Design Approach for High-Lift Systems incorporating Eccentric Beam Actuators
}

\author{
Durk Steenhuizen $^{1}$ and Michel J.L. van Tooren ${ }^{2}$ \\ Delft University of Technology, Delft, 2600, The Netherlands
}

\begin{abstract}
In order to asess the merit of novel high-lift structural concepts to the design of contemporary and future transport aircraft, a highly automated design routine is elaborated. The structure, purpose and evolution of this design routine is set-out with the use of Knowledge-Based Engineering techniques. As a case-study, the automated design of the Eccentric Beam Actuator is treated here, but the developed routine is meant to be applicable in a generic fashion, allowing for other concepts in the future.
\end{abstract}

\section{Introduction}

very lively field in current aerospace research is the reduction of environmental impact of future airliners. A This environmental impact can be described, on one hand, by the e.g. the amount of pollutants (e.g. NOx, C) or radiative forcing gasses $\left(\mathrm{CO}_{2}, \mathrm{H}_{2} \mathrm{O}\right)$ per passenger-km. On the other hand, next to pollution, the nuisance, which is most tangible as noise, that a community experiences from airplane operations is also an important factor as the demand for air-transport is increasing steadily. If future air-transport is to increase to meet its projected demand, nuisance in the form of e.g. the noise that an airplane produces in take-off and landing has to be reduced in order to adhere to airfield noise-regulations.

In order to reduce the environmental impact of future airliners, improvements can be made in the high-lift systems of the airplane. This is an obvious field of improvement where noise issues are concerned, as the predominant source of noise of a modern aircraft during landing, with engines idling, are the slots and sides of its deflected high-lift systems. Where pollutant emission is concerned, a reduction in cruise-flight drag and, hence, fuelburn, can be achieved by Natural Laminar Flow (NLF) technologies. A crucial requirement for NLF to be successful is a very smooth wing surface that does not exhibit any irregularities in the form of gaps and seams, which are formed by conventional stowed high-lift systems. Therefore smooth, seamless high-lift systems are an enabling technology for NLF to be successful.

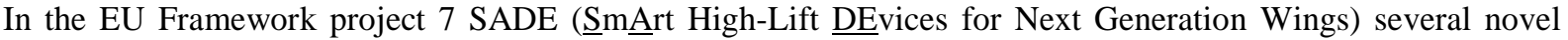
high-lift structural solutions are under investigation. These structural solutions have the potential to provide the required smooth, seamless high-lift devices, while at the same time reduce actuation power requirements and maintenance cost and reduce noise generation. The research goals of this project are a.o. to incease the Technology Readiness Level (TRL) of these high-lift technologies and produce knowledge with respect to their applicability to future airliner configurations.

\section{Detailed Formatting Instructions}

\section{A. Automated Design as a comparison tool}

To allow for a fair comparison between the different proposed structural solutions, whose performance improves that of current high-lift systems, actual comparative design studies have to be undertaken. By developing optimized preliminary designs of each novel high-lift concept, based on the same set of design requirements and constraints, a comparison can be made between these concepts with respect to a.o. weight, actuation energy and system complexity. Conclusions can be drawn on these results with respect to the best structural solution for a given application environment (i.e. the wing design, flight regimes, aircraft configuration).

By varying the input design requirements and constraints for this design exercise, conclusions can drawn on the suitability of the novel high-lift concepts for application to different airplane configurations. The current scope of

\footnotetext{
${ }^{1}$ PhD Researcher, Faculty of Aerospace Engineering, Kluyverweg 1, 2629 HS, Delft, AIAA Student Member.

${ }^{2}$ Professor, Faculty of Aerospace Engineering, , Kluyverweg 1, 2629 HS, Delft, AIAA Senior Member.
} 
this research is on the application to a conventional airliner wing, which is comparable in size and requirements to the Airbus A320 wing, but after initial design practices have been established and results obtained, this research will extend to other configuration, such as Blended Wing Bodies (BWB) and Prantl-Planes.

A challenge in assessing the applicability of the mentioned high-lift concepts is the low maturity of these concepts. Relatively little empirical design data exists for these concepts.

In order to facilitate the repeated design process of the various high-lift concepts, use is made of Knowledge Based Engineering (KBE) techniques. By employing KBE, repeating steps in the design process can be automated and hence can be repeated without too much effort. The development of a design tool is sought, which can, in a highly automated fashion, develop a feasible and optimized design solution of various novel high-lift structural solutions, for a given set of design constraints.

In order to implement a tool of the nature that is described above, the design of one actual implementation of a high-lift system that is considered in this research, is scrutinized and implemented in the code of the design routine. The design process that is thus created, forms a template for the design process of the other novel high-lift concepts that are considered in this research. The actuation concept that will be treated further here, is the Eccentric Beam Actuator (EBA).

\section{B. Determining the structure of the design process}

In order to arrive at a high-lift system conceptual design, a traditional outside-in design approach is taken, i.e. first the aerodynamic outer-surface is designed, and subsequently the internal structural details are 'filled-in'. This process is not fully performed in a serial fashion, as the aerodynamic design process usually does incorporate certain structural requirements for thickness and internal volume. These constraints serve to prevent aerodynamic design solutions being brought forward that would require an unwieldy internal structure.

In this design approach, should an undesirable structural design result after a sequence of aerodynamic and structural design processes, there is the option to reiterate this sequence of designs, whereby the structural constraints on the aerodynamic process are altered in a fashion that will likely result in a more desirable structural design solution (i.e. lighter, simpler, etc.). This design process can be considered as the the classical manual iterative design approach. Fig. 1 provides an illustration of this

On the other side of the spectrum of design approaches is the simultaneous multidiscliplanary optimization of both structural and aerodynamic designs. If an All-at-Once optimization structure is used, both design domains of the design in this example will be designed simultaneously, whereby the feasibility of the design is ensured by crosslinking constraints between both design domains. This approach is often considered to be the most appropriate for a multidisciplinary design optimization, as the subdomains of the design are all designed simultaneously, and none of these sub-domains will be conditionally optimal under the constraints imposed by a separate subdomain that follows from some previous design iteration. Hence, this approach will most likely arrive at the best possible global optimum for a given multidisciplinary design problem. The All-at-Once approach is depicted in Fig. 2.

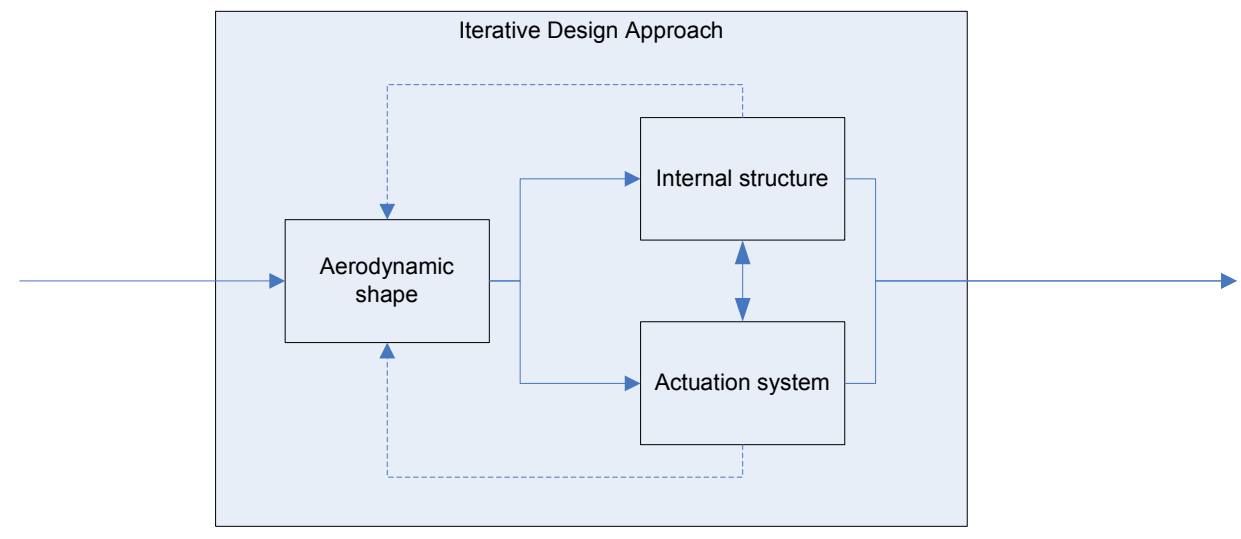

Figure 1. Representation of the Iterative Design process. 


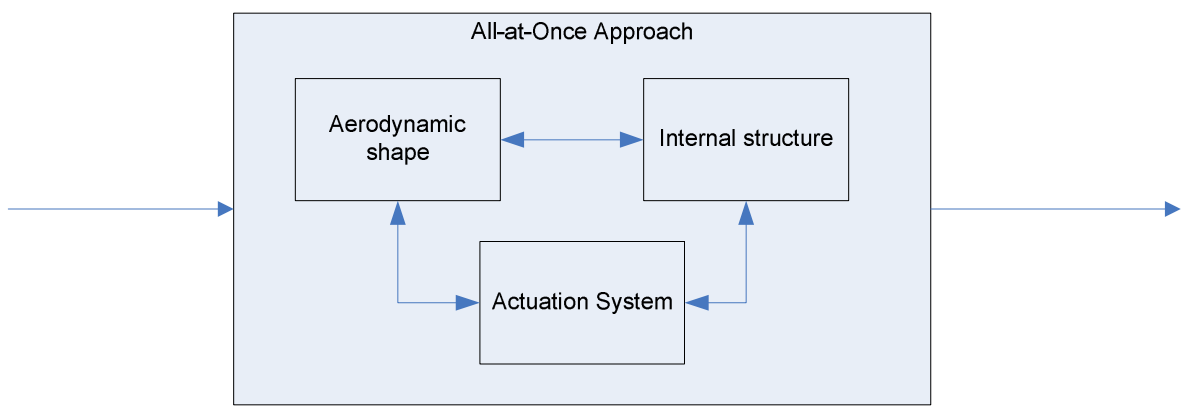

Figure 2. Representation of the All-at-Once optimization process.

Summarizing the differences between the classical iterative manual design and the All-at-Once MDO approach, it can be noted that the former approach uses far less computational effort than the latter, since the number of design iterations and aspect analyses is lower. On the other hand, the 'quality' of the resulting design for the manual approach is lower than for the All-at-Once approach, i.e. the latter method will likely produce a better design in terms of performance.

In order to set up an automated design approach for the high-lift systems treated in this paper, a compromise will have to be made between the two extremes of design approach that are described above. As the current research focuses on the conceptual design and initial design process, where multiple design concepts will be investigated, the preference is to keep computational effort for a given design to a minimum. The design process is therefore sequenced in a number of consecutive steps, where more detail is added to the design in each step. The strategy for this sequential design process is to make use of existing knowledge about the behaviour of the design with respect to its design variables and implement it in this process. For instance, knowledge of the behavior of the optimum solution of a simplified sub-problem of the design process can be implemented in order to bypass a computationally intensive optimization procedure.

The purpose of implementing this design knowledge into the design process is that it will reduce the number of design variables on which trade studies need to be performed. These trade studies serve to create various instantiations of the product in question that can be analysed with respect to their performance. If the combination of a number of variables that leads to best performance is known beforehand, a trade-study can be eliminated for these variables, thus quickly obtaining a feasible and optimum solution for a given sub-problem. In this fashion, the traditional feedback of data in order to reiterate, is replaced by straight throughput, at least for the sub-problems considered by pre-implemented design knowledge.

Where knowledge about a given sub-problem of the design is missing, an optimization routine of arbitrary complexity can be implemented to obtain a design solution. When such an optimization has to be performed many times, as is the case in the current research, it will prove beneficial to construct the required design knowledge for the sub-problem at hand. In that case, it will be useful to construct a response-surface of the performance-behavior with respect to the design variables of the problem at hand. This reponse surface will contain the optimization history of a number of optimizations, each with different constraint values, and will hence serve to interpolate a new design solution. In this fashion, a sub-optimisation in the design is replaced by an interpolation, thus saving computational effort. A precondition for this response-surface method to work is that the response-surface with respect to the design variables is sufficiently smooth, without any discontinuities, and that its domain covers all tobe-expected combinations of design variables.

In order to construct a response-surface of optimization results to a given design problem, the design-method that was developed up to that point in the sequence is a perfect candidate to supply the input design, that will have to be analyzed with respect to a given performance criterion. In this fashion the design tool that is under development is used to extend its own capabilities.

Next to implementing existing design-knowledge, optimization processes of various flavours and / or replacing these latter with response-surfaces, there is always the option of constructing a design approach by making educated guesses. This approach might be considered inacurate, but in a lot of cases this approach can generate a design solution that will not be too far off from the optimum solution. Especially in situations were the performance of the design has low dependence on the available design variables this approach will be beneficial. It is noted that care needs to be taken when using this approach, which involves the very human capabilities of common sense and educated guessing. For the current research this latter approach is used in design sub-problems were it is estimated that a variation in design parameters from the optimum combination will hardly produce a difference in the 
performance of the resulting design at the end of the sequence of sub-processes. While not completely accurate, this approach allows to save modeling effort, which can thus be focused on more demanding sub-problems.

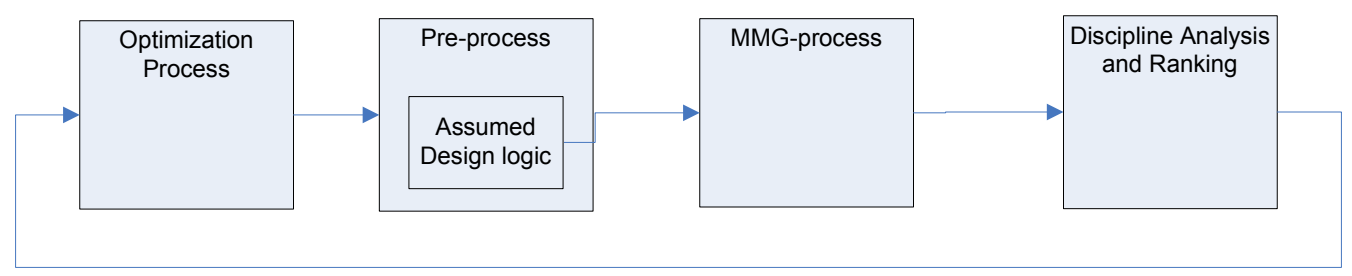

Figure 3. Implementation of the design process of a given design step in the procedure design. A certain design logic for some design variables is implemented, but still some design iterations must be performed.

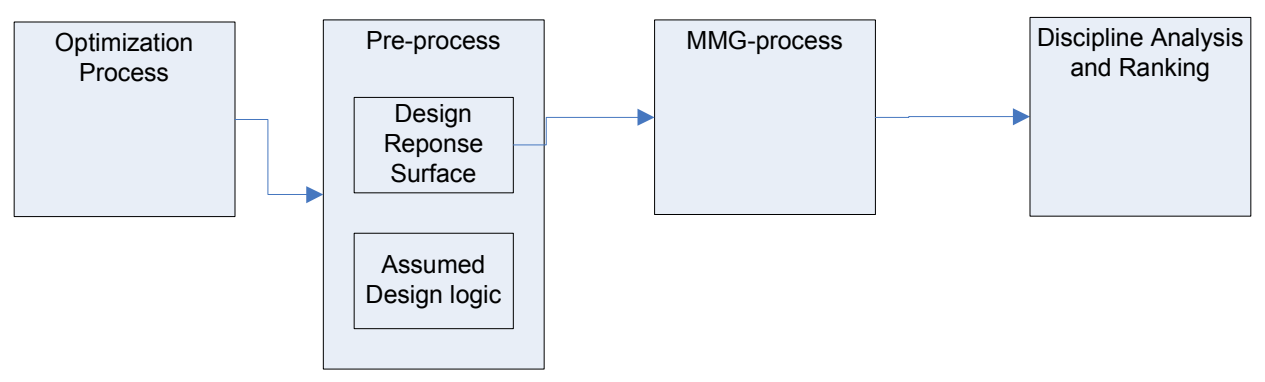

Figure 4. Implementation of the design process of a given design step in the procedure design with the implementation of design knowledge obtained earlier. A design response surface is implemented in order to allow input parameters to be directly translated into design-parameters.

The above-illustrated design process is illustrated by Figs. 3 and 4. Figure 3 depicts the situation where, for a given sub-design process, a certain design routine is assumed. In this routine the optimizer inputs a certain design vector $\mathrm{X}_{\mathrm{init}}$ into the design-preprocessor. The purpose of this pre-processor is to gradually reduce the number of design variables of the design vector $X_{\text {init }}$, in order to reduce the complexity of the design-optimization, which results in the reduced design vector $\mathrm{X}_{\mathrm{red}}$, containing the still free design-variables, and a derived design vector, containing the values that were derived from the implemented design knowledge. Fig. 4 adds a response surface to the preprocessor of Fig. 3 in order to allow for a quicker destermination of the optimum design solution. In the process, any feed-back loops to the pre-processor are eliminated.

The reduced and derived design vectors are input into a software-tool known as a Multi-Model Generator (MMG), which will construct a geometrical model of the product at hand, together with any discipline analysis models (e.g. FEM, Panel, CFD) for further analysis. These discipline models are subsequently analyzed by dedicated analysis modules and the interrelation of the various analysis disciplines is treated by a ranking routine, producing one or more performance indicators (e.g. weight, $C_{L} / C_{D}$, etc. ). These performance indicators are fed back to the optimization routine for the determination of the next design iteration.

The notions and considerations that are presented in this section are used to build a design tool known as a Design Engineering Engine (DEE). This DEE is a software tool, that is used to facilitate design processes of a generically defined class of products (e.g. an aircraft's wing). The DEE, in its general form, is built around an MMG, and performs the modeling, analysis and optimizations tasks described above. In addition the DEE is capable of identifying an initial estimate of the product design, in order to provide the optimizer with a good starting point. A structural overview of a generic DEE is given in Fig. 5.

The consecutive design-steps that are taken in order to arrive at a design solution for a given product, are schematically represented in Fig. 6. Here, the various steps in the design process are indicated by a set of consecutive iterations through the pre-processor, the MMG and the analysis routine. 


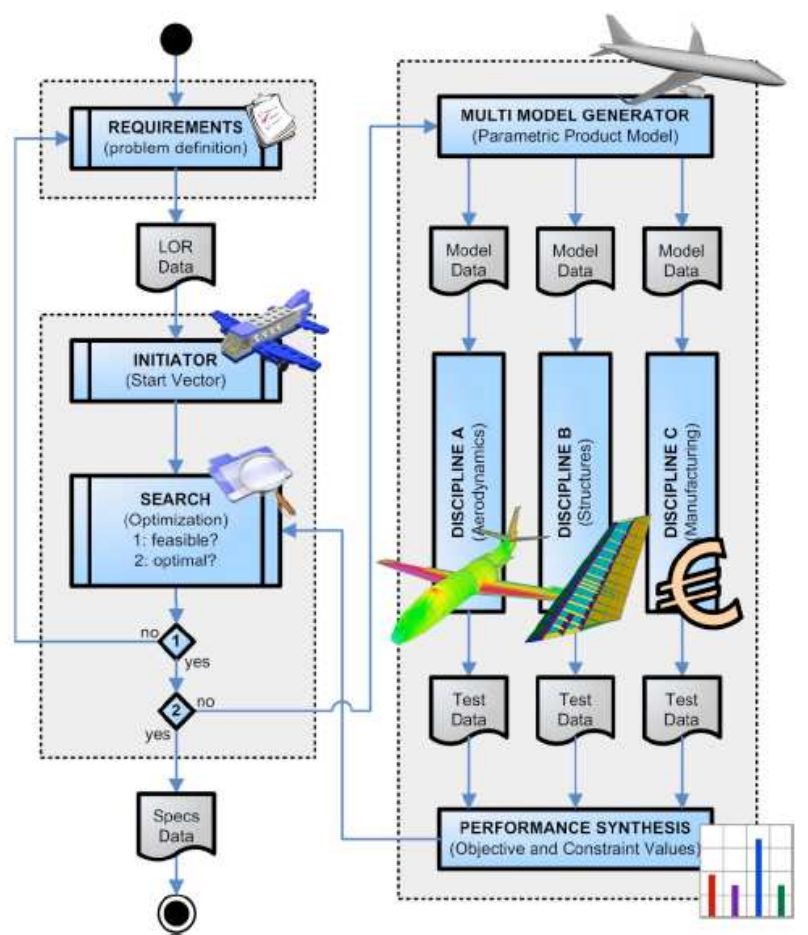

Figure 5. The Design Engineering Engine (DEE).

In this paper, an alteration to the general DEE concept is proposed, which will simplify the process of finding a feasible and optimal design for a given product. This alteration is comprised of a pre-processor to the MMG that will try to implement design-knowledge in order to reduce the number of design variables that need to be optimized.

By implying a design of certain features to be derived from a set of given input requirements, a number of the required variables that are needed to define the design, can be derived from these requirements. The task of the optimization process is to determine the performance behavior of the a given (sub-)design with respect to its principal defining variables. Once this knowledge is obtained, the response-surface can be used to synthesize (part of) the reduced design vector based on a given initial design vector.

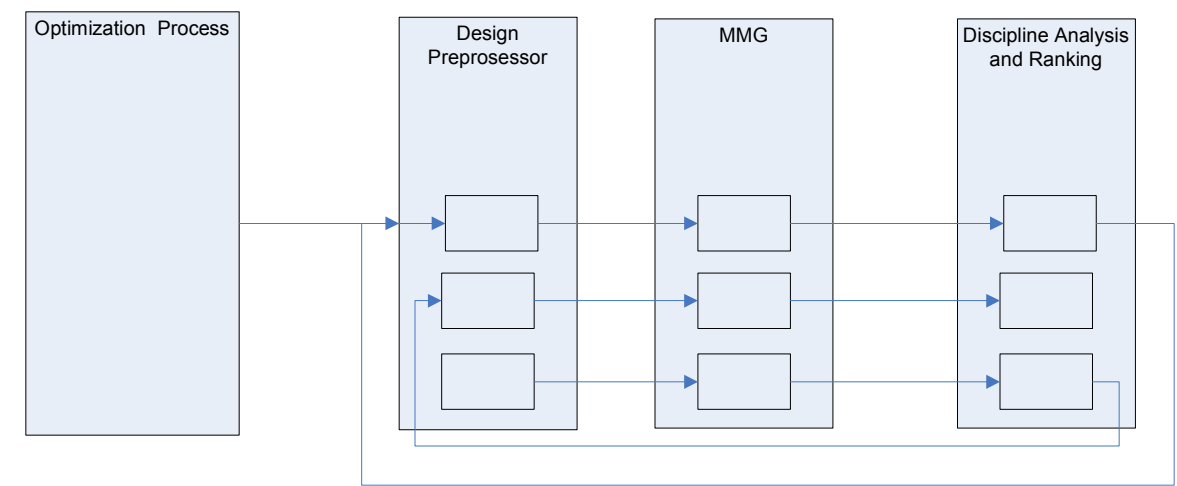

Figure 6. The design process of a high-lift system in its DEE structure. Various required consecutive steps can be identified that all have to perform a loop through the sequence of Design-Preprocessor, MMG, and Discipline Analysis modules. 


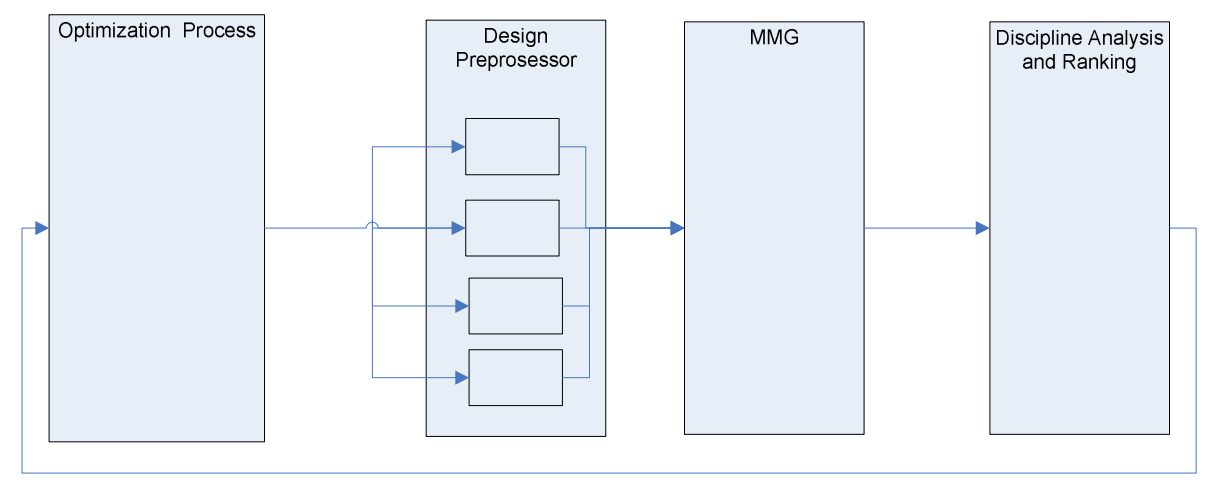

Figure 7. Design process of a high-lift system in its DEE structure when all available design-data has been obtained. By implementing this knowledge in the pre-processor a feasible and optimal design solution can be obtained for each combination of input parameters, thus precluding the need for an iterative process.

When zooming out from the sub-process level to the level of the complete design process, it can be noted that in order to construct a given product in a sequential fashion whereby more detail is determined in every step, one design sub-process has to be completed in order for the next to ensue. In order to find feasible, optimized solutions for each sub-problem of the complete design task, the MMG and the Analysis and Ranking functions have to be iterated many times, with the detail of the design increasing each time.

If all the required design-knowledge for each sub-problem is incorporated in the pre-processor, the initial design vector will only consist of the design requirements, which are subsequently translated by the pre-processor into design variables for the sizing of the product in question. These design variables are fed into the MMG, converted into aspect analysis models, and subsequently analyzed and merited.

The representation of a Design Engineering Engine with all possible design knowledge implemented would be a utopia, as it implies that all relevant knowledge is acquired. This situation is represented in Fig. 7. In practice, a useful DEE will be a hybrid between the two illustrated models, whereby some derived design variables are indeed determined by other input variables and requirements, while others need to be iteratively determined by some form of optimization routine.

\section{Eccentric Beam Actuator (EBA)}

An EBA in its most generic form consists of a rotating beam, whereby the axis of rotation does not coincide in part or in full with the central axis of the beam. By rotating such a beam, a shape change of the structure surrounding it can be achieved. EBA's have been successfully applied in the DARPA Smart Wing Project and considered as a candidate technology for future high-lift applications in the context of SADE. Specifically, the EBA that is considered in SADE is to be employed as a combined actuator and support structure of a flexible skin material in either the wing's leading or trailing edge sections. This flexible skin is initially considered to have a relatively low out-of-plane bending stiffness, while the extensional stiffness is very high. This results in a deformable skin that essentially maintains its cross-sectional arc-length.

The EBA itself is formed by a relatively slender, curved beam, with one end rotationally connected to the front spar of the wing-box, or to the rear spar in case of a trailing edge application. The other end of the beam is left free to move, with a variable number of cam-shaft-like discs positioned along its length. These discs serve to transmit forces between the beam structure and the flexible aerodynamic skin. They are considered to slide along the skin, while the disc-skin contact point remains on the skin surface, by some (to be implemented) constraining mechanism. Figures 8 and 9 show an example of an EBA applied to a flexible skin leading edge section. In both figures the shape of the beam and its discs is identical, the only difference being that it is rotated over 90 degrees about its left endpoint in the figure. 


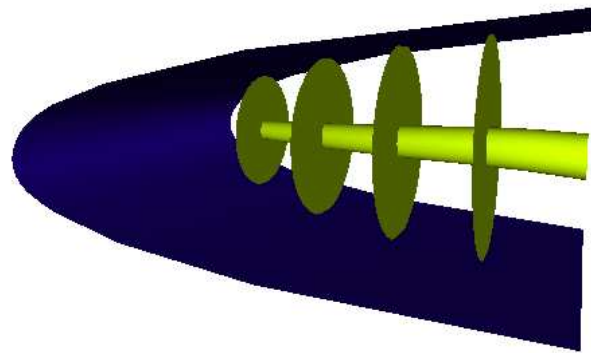

Figure 8. EBA-actuator in cruise configuration.

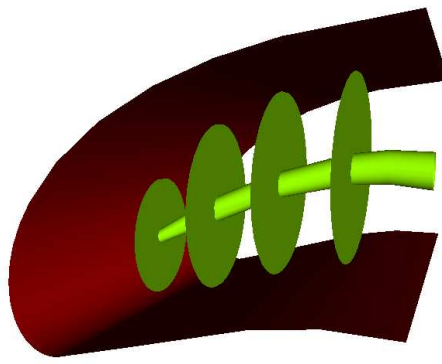

Figure 9. The same EBA actuator as before, but now in high-lift configuration, rotated over 90 degrees.

\section{Implementation of the EBA into an automated design routine}

\section{A. Multi-Model Generator (MMG)}

In order to be able to generate different instantiations of this EBA, a software tool known as a Multi Model Generator (MMG) is created. This MMG takes a set of descriptive input-parameters for the EBA and converts it to a geometric model of the intended EBA-design. Next to a geometric model, also structural analysis models (FEM), aerodynamic models and other disciplinary analysis models can be generated, based on the same set of input parameters.

The model that is used as the principal definition of the product to be designed is of a geometrical nature. All other definitions, such as aerodynamic and structural discipline models follow from this geometrical definition.

With the choices on structuring the design process, as set out in the preceding part, taken into account, a combination of preprocessor and MMG was developed for the design of an EBA.

A requirement of the EBA MMG is that it should produce a kinematicaly feasible EBA that does not violate any geometric constraint. To produce such a design, several assumptions are made by the MMG and a design approach is implemented, which is by no means the only method to arrive at a feasible geometric design, nor is it per se the method for obtaining the best design in every thinkable situation. It is assumed here that variations in the implemented geometrical design process produce little variation in the final preliminary high-lift system design.

Without going into too much detail here about the geometrical design procedure employed, the EBA MMG takes as input the two aerodynamic surface configurations (e.g. the cruise and high-lift configurations) together with the number and positions in chord-wise direction of the discs. Also material thicknesses and properties can be input for the beam and its discs.

Now, the disc contact points with the skin are assumed to move only in the spanwise direction on the skin's surface as the EBA rotates between its two extreme states. More specifically, this means that the arc-length of the distance between the contact-point and the LE (or TE) spar taken along the skin remains equal. Because of the deformation of the LE (or TE) while the EBA is rotated, the above-described contact-points move not only in an updown direction (parallel to the airplane's z-axis), but also in the chordwise direction.

Another assumption implemented in the MMG is that the beam's projected shape, when viewed in cross-section, should run along the local camber line of the input-surfaces in both extreme deflection positions of the beam. Because the beam is assumed to rotate over 90 degrees, these two described projected shapes are orthogonal to each other and together uniquely describe the 3D-shape of the beam.

With a beam defined according to the above design assumptions, the discs are placed along the beam according to the chordwise input-positions. To accommodate for the chord-wise movement of the disc-skin contact-points, the disc are shaped as non-planar ellipses.

The EBA's that are obtained by the said MMG still have to be designed with respect to their structural properties. Furthermore, the position and number of the beams have to be optimized as well. Thus, the MMG is used in a structural optimization-loop that will determine the said variables in order to minimize structural weight, actuation energy, or a compound objective function of these two with aero-elastic deformation as a side-constraint next to the obvious constraint that the EBA-must be strong enough to carry the imparted loads safely.

The aero-elastic deformation is chosen as a side-constraint, since it has a large influence on the aerodynamic performance of the high-lift system, but behaves as a system with a certain threshold. That is, the forward movement of the BL transition point can handle a certain amount of LE shape deformation before it will actually start accelerating abruptly and rapidly with reducing skin-stiffness. The skin's stiffness has to be reduced as compared to 
a regular rigid skin in order to be able to deform without too much undue actuation force. If the stiffness becomes too low, the skin will start to deform noticeably under aerodynamic loading, altering the airfoil's shape and, hence, degrading performance. There will therefore be a certain influence of the laminar flow requirement on the stiffness properties of both the EBA and the skin that it is supporting.

Giving the above considerations, it is to be expected that lowest weight and actuation energy are obtained when the skin-stiffness value approaches the threshold value determined by the migration of the transition-point.

It is envisioned that a feasible and optimal combination of the mentioned variables will be obtained from a suitable optimization process. It is therefore decided to use the MMG-structure that is developed up to this point, in order to investigate the design behavior of the EBA and the nose skin-surface. The goal of this is to develop knowledge in fashion mentioned above in order to extend the design tool.

\section{B. Design of a smooth, seamless high-lift aerodynamic surface}

Since this study focuses on the application of novel high-lift structural concepts to existing and future aircraft configurations, the overall aerodynamic and structural design of the wing fall outside the scope of the to-be developed design tool and are therefore considered as input to the design process. This design tool will rather focus on the design of the actual high-lift systems and how they are applied on a predefined wing configuration.

The aerodynamic design of the leading edge part of the high-lift system consists of generating a smooth, seamless aerodynamic surface. The first step in this process is to specify the contour of the LE high-lift system on the input wing in cruise configuration. Based on this contour, the cruise-configuration surface of the LE device is cut-out of the wing and stored for further processing. This process is illustrated in Fig. 10.

An arbitrary number of 2D streamwise cross-sections can be taken of the wing surface over the section with the now cut-out nose section. To each of these cut airfoil sections, a new high-lift nose part is fitted, as to obtain a new closed high-lift airfoil. The airfoils that result in this fashion are used to construct a 3-dimensional surface for this high-lift LE part.

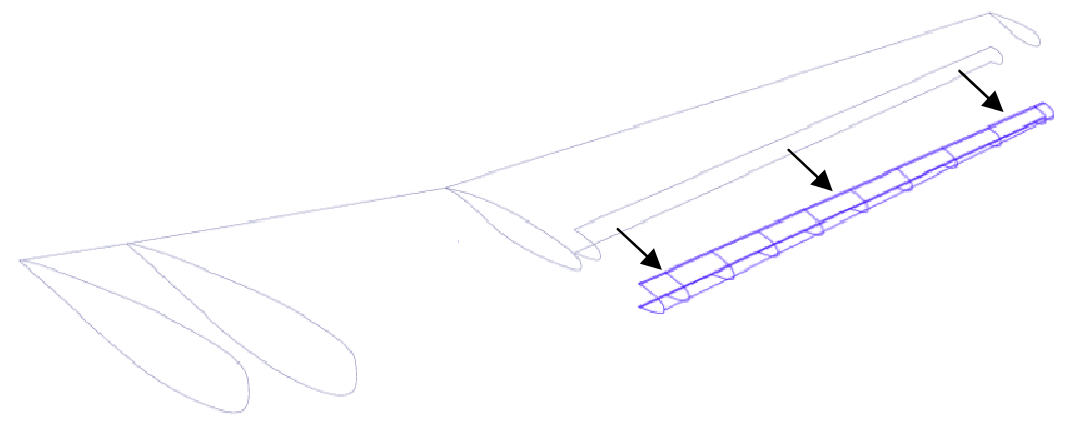

Figure 10. A wing aerodynamic surface is provided by the MMG, based on fixed input parameters. A segment of this wing is cut at the location were a high-lift system is to be fitted. The cut-out surface is stored to serve in the ensuing design process of the high-lift system

The shape of the new high-lift nose airfoil sections has quite a lot of freedom of design. Some considerations for the design of this nose section are its arc-length and the degree of continuity at the connection points with the original cut airfoil. For novel skin constructions that can stretch and contract in-plane, a high lift nose section can be chosen with an arc-length different from that of the original cruise nose cross-section. For skin constructions that are not meant to stretch and obtain their deformability from out-of-plane bending, the high-lift cross-sectional arc-length should be taken the same as that of the original cruise cross-section.

Where the degree of continuity at the connection is concerned, there should obviously be at least a C0continuity, to avoid any seams or slots in the airfoil, C1-continuity also helps in improving the boundary-layer flow at these intersections, but in order to achieve a continuous pressure coefficient $\left(\mathrm{C}_{\mathrm{p}}\right)$ distribution across the connecting points, $\mathrm{C} 2$-continuity, i.e. a continuous radius of curvature distribution, would be required.

While the aerodynamic shape of the above described high-lift airfoil sections is a prime candidate for an optimization process, it is for the current design tool taken as a fixed input-shape, which was obtained from a separate and dedicated 2D optimization process. This cross-sectional shape assumes no in-plane stretching. Furthermore, it uses C2-continuity for the upper LE-to-main airfoil connection, to prevent any unwanted pressure- 
jumps on the boundary-layer flow in that region. For the bottom connection point, only $\mathrm{C} 1$ continuity is imposed, as the boundary layer flow is much more favorable in this region.

With the LE high-lift cross-sections obtained in the fashion described above, these sections are subsequently used to construct an aerodynamic surface by means of a geometrical routine, known as lofting. For the current highlift system, use is made of a linear loft between two LE cross-sections, which are positioned at either spanwise end of the deflected LE nose-section.
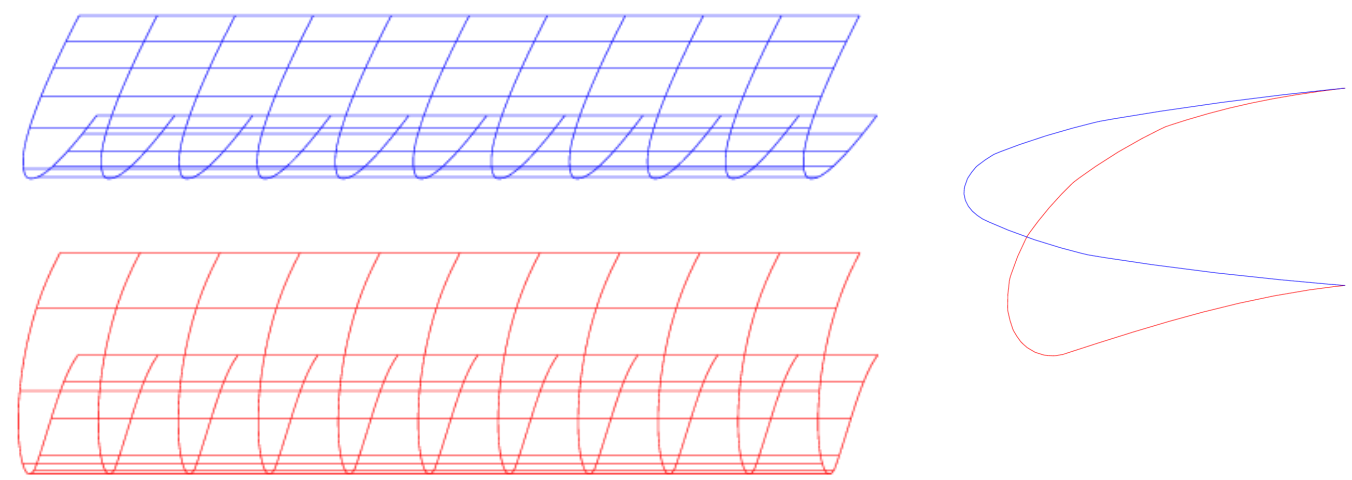

Figure 11. Input surfaces for the design routine for a TE-applied EBA.

Now that an initial estimate for the high-lift LE aerodynamic surface is obtained, this surface still does not fully adhere to the imposed top-level design-requirement of a seamless surface. If a traditional solution were pursued, the spanwise edges of the LE-device would form local discontinuities to the aerodynamic shape of the whole wing. These discontinuities will persist as streamwise seams in the retracted LE configuration and will hence function as a source of boundary-layer disturbance, which will expedite BL-transition, thus precluding NLF. In the high-lift configuration the edges that are formed give rise to airflow leaking around them, which causes turbulent vortices that contribute to approach-noise. Fig. 11 gives an overview of the relevant input-surfaces to the current design problem.

Taking these notions into consideration calls for a smooth blending between the deflected high-lift LE surface, which was previously obtained, and the otherwise undeformed rest of the wing. A code that performs this task was developed and implemented in the design tool.

This spanwise blending routine takes the preliminary high-lift LE surface as input and slices it up into three parts. These parts are the 2 to-be-blended edge sections, which arise as the adjoining wing section is to be left unaltered. The third and middle section is the portion of the high-lift surface that is to be left unaltered.

The smooth blending surface between the edges of the third LE section and the spanwise edges of the unaltered wing are constructed by making use of a geometrical construct known as an edge-blend-surface.

This edge-blend-surface is constructed by considering both the edges between which a blending-surface is to be constructed and to adjoining surfaces that it will connect. The routine that constructs this blend considers the input edges and determines the adjoining surface's directional derivative in a direction locally perpendicular to the edgecurve and does this along the length of the edge-curve. The blending routine will construct a blend based on intersecting cross-sectional contour-curves that are defined in an orthogonal reference frame, termed the surface's $u$ and $\mathrm{v}$ direction. The chordwise contour-curves $(\mathrm{u})$ of this blend are formed by interpolating between the adjacent wing's edges. The spanwise contour curves (in the v-direction) are constructed by $3^{\text {rd }}$ degree Bezier curves.

A Bezier curve's shape is most easily influenced through the position of its control points. Some properties of the Bezier curve is useful in the creation of aerodynamic surfaces. Irrespective of the degree of a given Bezier curve, its end-points $\mathrm{P}_{0}$ and $\mathrm{P}_{\mathrm{n}}$ will always be interpolated at the edges of the curve's parametric space (usually [0, 1]) which opens the possibility to impose end-point values to the curve at hand, i.e. impose $\mathrm{C} 0$-continuity. Furthermore, it turns out that the directional derivative at the end-points are equal to the direction vector that is formed between the end-point of a curve's control-point grid and the point immediately adjacent to this point, i.e. between $\mathrm{P}_{0}$ and $\mathrm{P}_{1}$ for the starting-point and $\mathrm{P}_{\mathrm{n}-1}$ and $\mathrm{P}_{\mathrm{n}}$ for the end-point. This notion is used to construct a curve that has $\mathrm{C} 1$ continuity with adjacent curves at its end-points.

With the two conditions for $\mathrm{C} 0$ and $\mathrm{C} 1$ continuity at the curve's end-points known, it is observed that in order to obtain a Bezier curve that connects to both extreme points' ends in a $\mathrm{C} 1$ fashion, the set of points $\left[\mathrm{P}_{0}, \mathrm{P}_{1}, \mathrm{P}_{\mathrm{n}-1}, \mathrm{P}_{\mathrm{n}}\right]$ is required. The simplest Bezier curve that shows adherence to this requirement is a curve that is defined by four 
control-points, i.e. it is of $3^{\text {rd }}$ degree. It is exactly this curve that is used to determine the desired contour-curve, in the v-direction, for the blend surface. An example of a blended LE high-lift surface is depicted in Fig. 12.

When placing the control points, while adhering to the above-mentioned $\mathrm{C} 1$-constraints, the designer is still left with 2 extra degrees of freedom in the curve's definition. While the endpoints $\left(\mathrm{P}_{0}, \mathrm{P}_{\mathrm{n}}\right)$ and the placement direction of the points immediately adjacent $\left(\mathrm{P}_{1}, \mathrm{P}_{\mathrm{n}-1}\right)$ are known, the distances by which the adjacent points should be offset from the endpoints are free to choose. This freedom of design does not violate the imposed continuity constraints. These offset distances are subsequently used as input-parameters in the aerodynamic design process, together with the widths of the blend-sections. In order to introduce a non-dimensional parameter for these offset distances, that only influences the shape of the curve, irrespective of its magnitude, they have to be normalized with respect to a conveniently chosen dimension. This dimension is chosen as the direct distance between both extreme points. Thus the blend-ratio is introduced for both inboard and outboard LE high-lift surface blends. This ratio is defined as the offset distance of an adjacent point with respect to its end-point divided by the distance between both end-points.

Variations in the aerodynamic performance behavior of both the edge-blend-surfaces need to be studied. It is foreseen that when the width of the blends is too small, the BL will be very sensitive to separation. Also, the exact shape of the blend (governed by the edge-blend-ratios) is considered as influential, but of lower order.
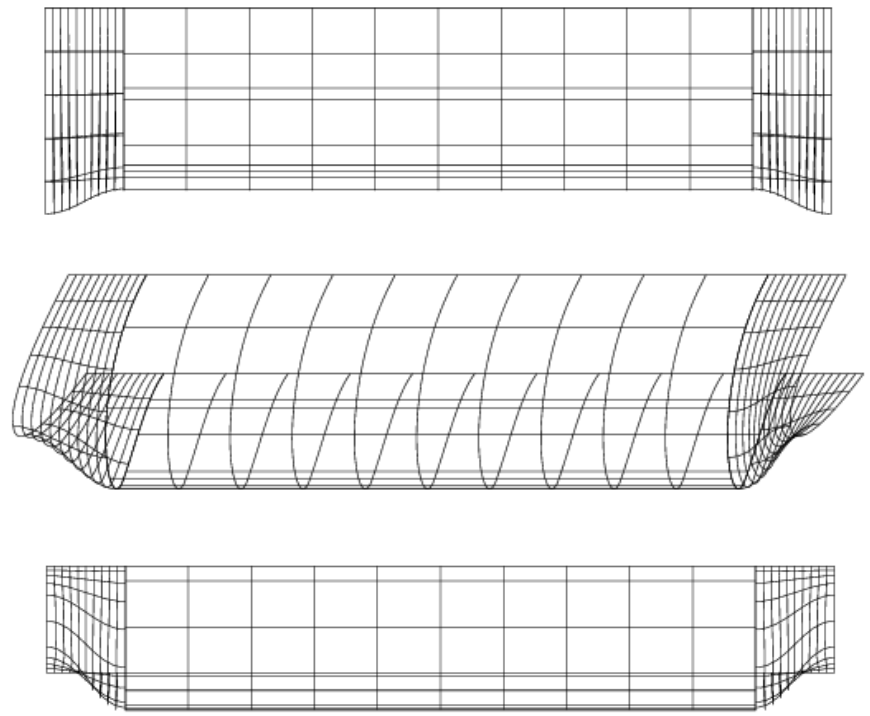

Figure 12. Resulting LE surface after blending of the edges, shown respectively top-down, tri-metric, and front-to-rear.

\section{Development of a smooth variable camber tail section}

Another focal point for this research is the applicability of the novel high-lift devices to the trailing edge of a wing. An example of such an implementation is provided in Fig. 13. In this region of the wing, the aerodynamic requirements with respect to NLF are less stringent, as the flow will have passed most of wing's chord, so a tripping of the BL into transition in this region will have less dramatic effects on the cruise drag of this wing. Moreover, it is quite possible that the BL flow will have already tripped upstream from this TE section, in spite of the NLF design implementations to the wing. In this case, the skin-stiffness requirements of the TE device can be relaxed to some degree.

In this part of the high-lift system design, again an aerodynamic optimization would be appropriate. In fact, since the LE and TE high-lift devices have a large influence on each other's working and on the overall high-lift performance of the wing, the best optimization approach would be to simultaneously optimize the shape of both the LE and TE parts, at least for one or several 2D-sections of the high-lift wing.

In the context of this research, such an optimization was performed separately of the current design tool development, and a high-lift TE cross-section was provided. This TE section naturally belongs to the LE crosssection that was provided earlier for the LE section construction. It was decided to drop the requirement of a seamless device at the TE, since this requirement would preclude the possibility of constructing a multi-element airfoil, which would pose a large inhibition on the achievable maximum-lift of this device. By allowing a single 
slotted flap configuration, a compromise between system complexity and high-lift performance was arrived at. This stipulation is observed in the development of the high-lift design-tool.

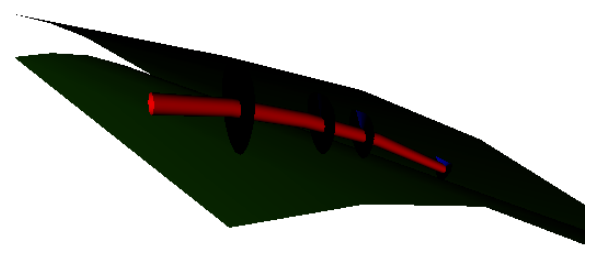

\section{Figure 13. EBA applied to a variable camber TE section of a wing}

A novel application for the considered high-lift configurations is the application of camber trimming of the wing's cross-section during flight. While an aircraft cruises at it's cruising altitude, its weight will reduce as the flight progresses. While weight reduces, so does the required lift to keep the aircraft in level flight. There are three distinct variables available to adjust the wing's lift to the reducing weight. The first of these is a reduction in airspeed, which is generally undesirable, because this will increase the aircraft's block time. The second approach is to reduce the air density by increasing the cruise altitude steadily as the cruise progresses. While very feasible in theory, practice learns that current air-trafic operations generally don't allow such a direct cruise-climb flight profile and an actual cruise-profile will consist of a stepped-cruise, where each step in the altitude is coordinated with ground-control. Another issue with this approach is that for a turbofanned airliner the required fuel to cruise a given distance decreases with air-density and hence with increasing altitude. This implies that the best fuel-saving strategy will be to cruise at the highest-possible altitude, leaving little room to climb during cruise.

The third approach is to change the $\mathrm{C}_{\mathrm{L}}$-value that the wing needs to provide. The implication of this is that with this $C_{L}$-value the wing will also achieve a different, and likely lower, $\left[C_{L} / C_{D}\right]$. In a proper aircraft sizing study the wing is generally designed to provide optimum $\left[\mathrm{C}_{\mathrm{L}} / \mathrm{C}_{\mathrm{D}}\right]$ at the cruise $\mathrm{C}_{\mathrm{L}}$ needed for the designed cruising airspeed, altitude, and wing-loading W/S. Therefore, a device that can alter the wing's $\mathrm{C}_{\mathrm{L}}$-value at which it's maximum $\left[\mathrm{C}_{\mathrm{L}} / \mathrm{C}_{\mathrm{D}}\right]$-value is achieved, without a significant loss in the actual magnitude of this ratio, is sought. A solution to this requirement is to adjust, or rather, 'trim' the camber of the wing's cross-section, which will adjust the optimum $\mathrm{C}_{\mathrm{L}}$-value in the fashion described. Studies on airfoils with different camber-settings show that the second requirement, on $\left[\mathrm{C}_{\mathrm{L}} / \mathrm{C}_{\mathrm{D}}\right]$, can be fulfilled. It is considered an interesting goal to study the ways in which such a device can be implemented structurally.

In order to trim the camber of the wing's chordwise cross-section, a promising approach seems to be to deform only part of this shape. A common approach is to adjust the shape of the LE, located past, say, $70 \%$ of the chord. For this study such devices will be investigated at a preliminary design level and the design engine will be adjusted accordingly to provide the modeling and analysis of the design. While a variable camber TE section can be most easily implemented by in-plane stretching of the aerodynamic skin, for the moment a design sub-process was implemented that assumes that the skin is relatively stiff in the in-plane direction and a variation in the TE crosssection is mainly caused by out-of-plane bending of the skin panels. To allow the upper and lower skins of such a TE device to deflect, they should be allowed to move with respect to each other. Therefore, in the current implementation it is decided to leave the TE edge of this device open, without connecting the upper and lower skin in a rigid fashion. By allowing the skins to slide with respect to each other, a variable camber is achieved. It will be assumed that some form of seal is implemented at a later stage in the design process, between the sliding upper and lower TE surfaces.

As a parameterization for this LE device, the upper and lower surface TE parts are defined as b-spline curves. The shapes of these curves are influenced by the respective control-points of both curves, which are in turn defined in a fashion relative to the camber-line of this LE section. By adjusting the camberline shape, the location of the control-points is adjusted as well. The parameterization routine takes into account C2-continuity at the connection points with the main (rigid) airfoil-section. In addition, a scaling routine ensures that the arc-length of these altered curves remain identical to the original arc-lengths. The shape of the camberline is defined by a 3rd degree Bernstein polynomial. The first 2 of the total of 4 shape parameters of this curve are used to attach the constructed TE camberline to the remainder of the original camberline in a $\mathrm{C} 1$ fashion. This leaves a total of 2 shape parameters to optimize the deflection of the TE. 
In order to determine the optimum LE surface contour for a given $\mathrm{C}_{\mathrm{L}}$-setting, a genetic optimization was undertaken, based on the modeling provided by the design engine. For this optimization a genetic algorithm was selected. The initial cruise and optimized camber-trimmed-airfoils are shown in Fig. 14, the resulting blend of the two surfaces is depicted in Fig. 15.

Now that a routine is obtained to develop a camber-trimmable wing for the plain cruise airfoil, a more realistic design will be implemented in the future for an EBA that is located in the single flap of the high-lift configuration. By implementing variable camber in this part, the retracted cross-section of the wing will also inherit its variable camber properties for the rear part of the cruise-airfoil. This "upgrade" to the design process, however, will not be elaborated further here.

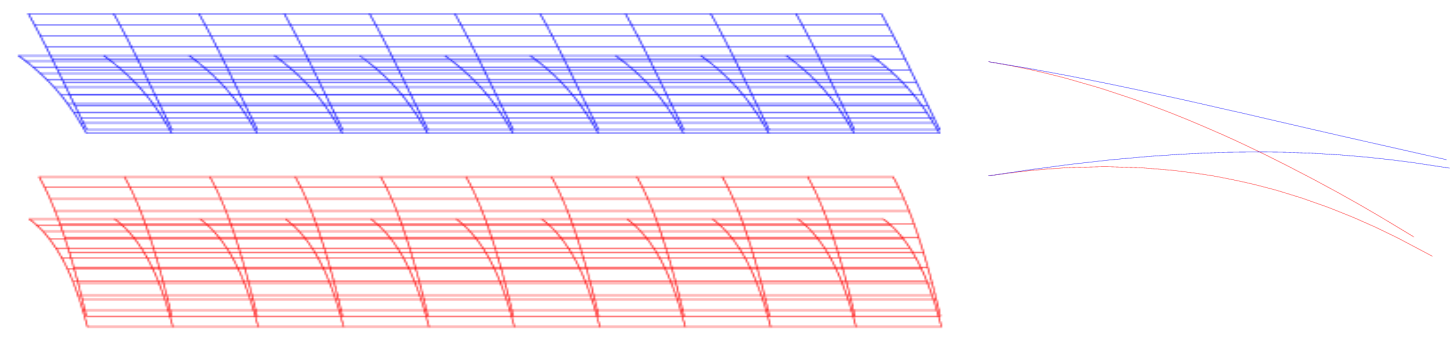

Figure 14. Input surfaces for the design routine for a TE-applied EBA.
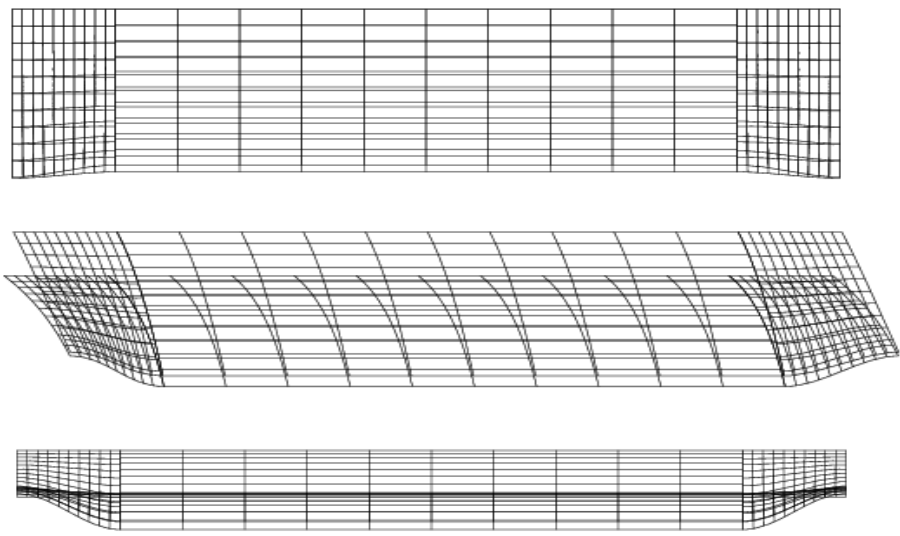

Figure 15. Resulting TE surface after blending of the edges, shown respectively top-down, tri-metric, and rear-to-front.

\section{Summary of the obtained design structure}

With part of the design process of an EBA structured in the fashion that is described in the preceding sections, the design structure will be summarized here.

The first step in the design process is to make smooth blending surfaces for both the LE and TE sections, for both high-lift and camber trimming applications of the EBA. As input to this step, the cruise skin surface is obtained from the pre-defined wing surface. The high-lift surface is obtained by lofting a set of pre-defined high-lift nose sections.

In order to make an effective blend between the high-lift device and the rest of the wing, the breath and the shape of the blends must be optimized. This process is illustrated in Fig. 16 where a feed-back is implemented from the aerodynamic analysis routine back to the pre-processor. This optimization will be performed to obtain the best shape of the blending sections required. Since most of the input data at this point is fixed, it will come as no surprise that a single optimum shape will be obtained. For the current research, this outcome is acceptable and the blending process can subsequently be implemented as a straight through-put, whereby the known optimum blend shape is applied to the input cruise and high-lift surfaces. This blended surface is used downstream in the design process to start the sizing of the EBA's. 
In order to further develop this step in the design-process, the optimization of the blend, in the fashion described above, has to be performed multiple times for different input surface shapes. In this fashion, a response surface of the optimum blend shape will be obtained for implementation in the design routine. This situation is illustrated in Fig 17.

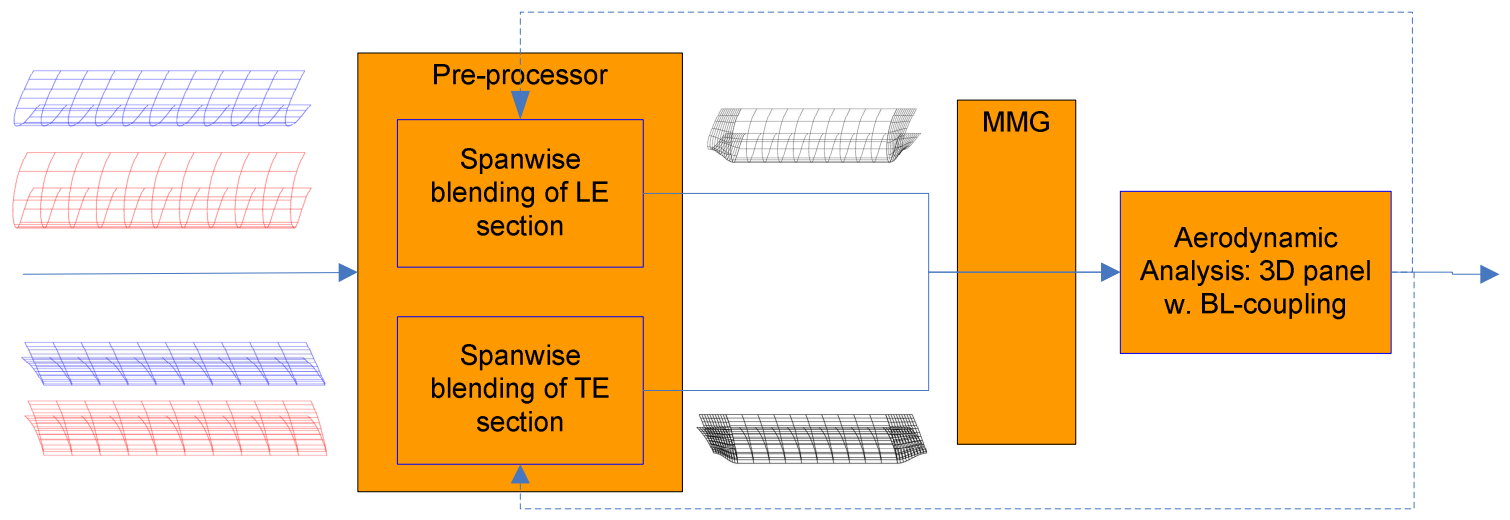

Figure 16. Layout of the design process for the blending of high-lift system's edges when no previous knowledge on the optimization behavior exists.

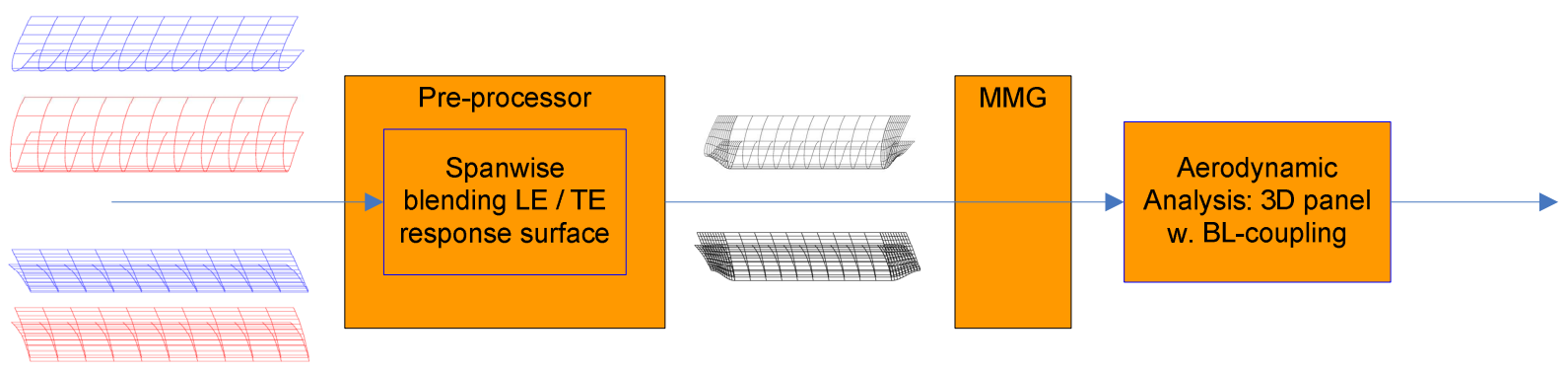

Figure 17. Layout of the design process for the blending of high-lift system's edges when a response surface has been implemented.

With smoothly blended LE and TE surfaces obtained from the previous design step, these will be subsequently used to design the actual EBA's that need to support and actuate these surfaces. As was indicated before, the design of the EBA's can be divided into roughly 3 parts, being the kinematic sizing of the beam and its discs, the sizing of the stiffness of the beam, and the sizing of the skin stiffness distribution. The first of these, the kinematic sizing is implemented in a straightforward fashion, whereby most dimensions are deduced from the input cruise and high-lift surfaces. The main variables of importance to the optimization of the rest of the high-lift system's design are the number and location of the actuating discs that support and move the skin. The kinematic sizing routine that was previously elaborated is implemented in the design routine of this design step.

The sizing of the beam's and skin's stiffnesses are highly dependant on the number and location of the discs. These variables are a determining factor for the introduction of bending loads into the beam, while at the same time the skin's stiffness requirements are predominated by the unsupported distance that the skin needs to traverse between two adjacent disc's contact points. In order to prevent detrimental aerodynamic effects, the amount of sagging or bulging of this unsupported skin must be kept within certain bounds.

Initially, the above-stated considerations for the design process of this step are implemented in a traditional manual-like fashion. This is indicated in Fig. 18 a number of reiterations will likely have to be taken in order to develop a feasible, let alone, optimal design solution. Therefore some kind of combined optimization routine is to be developed in order to automate this process. In Fig. 19 this implication is illustrated by an All-at-Once optimization structure. It is stretched here that this optimization structure is by no means the only or the best approach. Actually, a Collaborative Optimization (CO) is sought for implementation. The benefit of this approach is that the required iterations are reduced at the expense of possibly obtaining a slightly sub-optimal solution. 
The ultimate goal in the construction of the current design step is to obtain a response surface of the optimization results of many different combinations of input variables, such as the operating aircraft's wingloading W/S and input-surfaces. By implementing such a response-surface, a quick routine, with a minimum of feed-back is obtained for this design step. This (still to be implemented) process is depicted in Fig 20.

This section has presented an initial lay-out of the chosen approach in developing a software tool to automate the design process of novel high-lift structural solutions. The purpose of this will be to allow for comparative design studies of a number of proposed concepts. The design process developed here is far from complete, but is set up in such a fashion that knowledge about its behavior can be put to good use, allows for improvements as more knowledge is obtained, and is modular and portable so that it can be recycled for other, similar, studies.

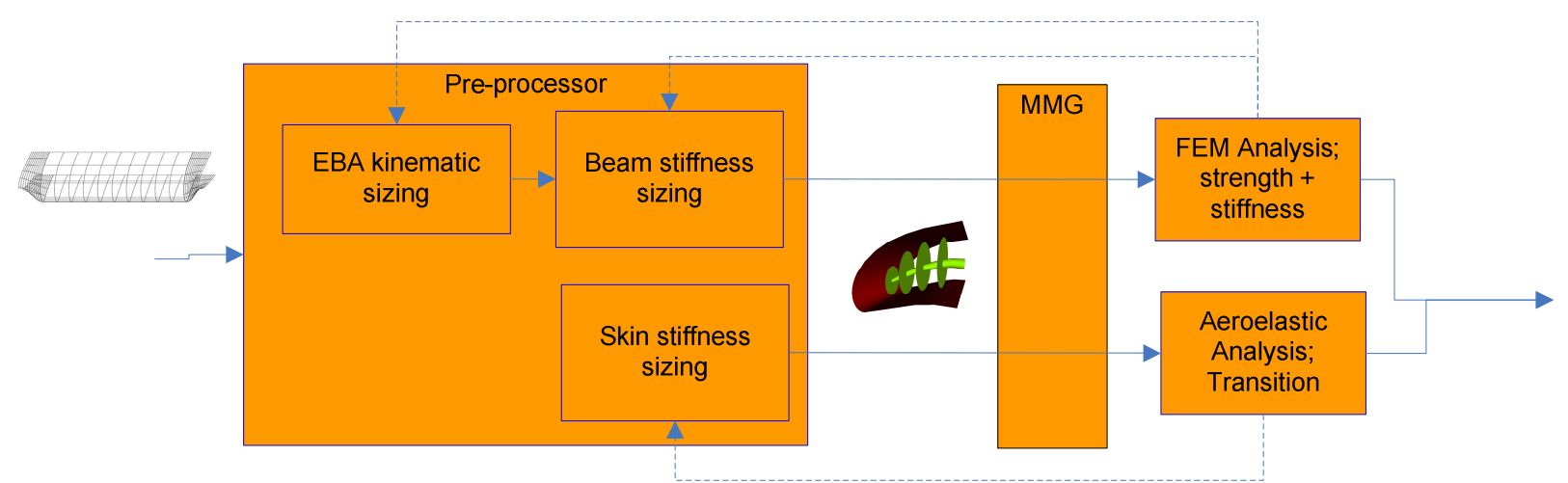

Figure 18. Layout of the design process for the EBA when no previous knowledge on the optimization behavior exists. Several iterative loops are implemented in this process.

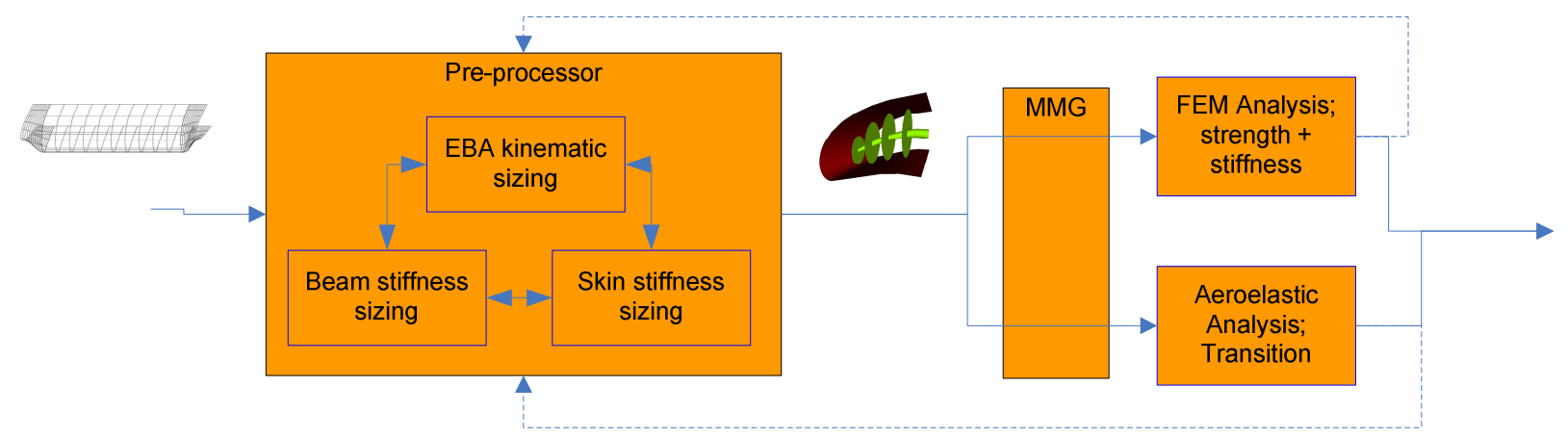

Figure 19. Layout of the design process for the EBA with the inclusion of some form of multi-system optimization process. This process will autonomously obtain an optimum design solution for a given set of input-parameters.

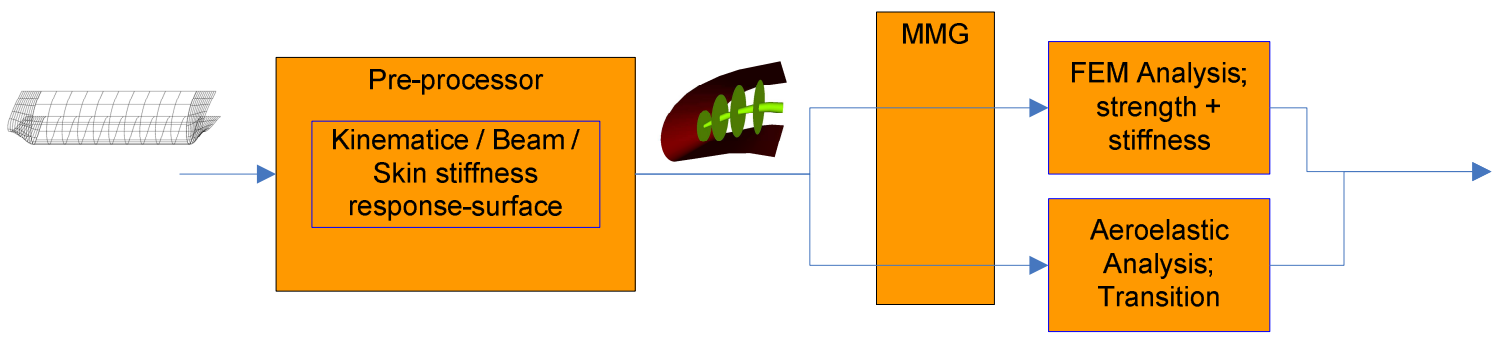

Figure 20. Layout of the design process for the EBA when a response surface has been implemented. 


\section{References}

J. D. Bartley-Cho, D. P. Wang, C. A. Martin, J. N. Kudva and M. N. West, "Development of High-rate, adaptive Trailing Edge Control Surface for the Smart Wing Phase 2 Wind Tunnel Model”, Journal of Intelligent Material Systems and Structures, Vol.15, April 2004.

C.L. Emberey, N.R. Milton, J. Berends, M. Van Tooren and S. Van Der Elst, B. Vermeulen, "Application of Knowledge Engineering Methodologies to Support Engineering Design Application Development in Aerospace", 7th AIAA Aviation Technology, Integration and Operations Conference (ATIO), AIAA-2007-7708, Belfast, Ireland, 2007

G. La Rocca, M.J.L van Tooren, "A Knowledge Based Engineering Approach to Support Automatic Generation of FE Models in Aircraft Design", 45th AIAA Aerospace Sciences Meeting and Exhibit, AIAA-2007-0967, Reno, NV, USA, 2007

La Rocca, G. and van Tooren, M.J.L., "A modular reconfigurable software tool to support distributed multidisciplinary design and optimisation of complex products", 16th CIRP International Design Seminar, Kananaskis, AB, Canada, 2006

C. Waldhart, et. al., Analysis of tow placed, parallel fiber, variable stiffness laminated, $37^{\text {th }}$ AIAA/ASME/ASCE/AHS/ASC Structures, Structural Dynamics \& Materials Conference, 15-17 April, Salt Lake City, UT.

M.H.Love, P.S.Zink, R.L.Stroud, D.R.Bye, C.Chase, Impact of Actuation Concepts on Morphing Aircraft Structures, AIAA 2004-1724

D.Steenhuizen et. al., "SADE - Database of Smart Activation Principles with Available Performance", internal report $7^{\text {th }}$ Framework Project SADE, Delft, Jan. 212009.

J. Schut, M. van Tooren, J. Berends, "Feasilization of a structural wing design problem", 49th AIAA/ASME/ASCE/AHS/ASC Structures, Structural Dynamics, and Materials Conference, AIAA-2008-2142, Schaumburg, IL, Apr. 7-10, 2008.

M. Perera and S. Guo, "Optimal design of an aeroelastic wing structure with seamless control surfaces", Proceedings of the Institution of Mechanical Engineers, Vol 223 Part G: Journal of Aerospace Engineering, 9 July 2009.

P. Y. Papalambros and D. J. Wilde, "Principles of Optimal Design", $2^{\text {nd }}$ ed., Cambridge University Press, ISBN 0-52162727-3, Cambridge, 2000. 